\title{
Numerical validation of microwave heated fluidised bed calcination of waste containing ceramic powders
}
V. Vegh*
I. W. Turner ${ }^{\dagger}$
E. Sizgek ${ }^{\ddagger}$
D. Sizgek S $^{\S}$

(Received 1 August 2003; revised 8 January 2004)

\begin{abstract}
This study corresponds to the first phase of the development of a microwave heated fluidised bed for calcination of waste (metal nitrate salts) containing ceramic powders under $\mathrm{H}_{2} / \mathrm{N}_{2}$ reducing atmosphere. The power is delivered through the top end of the calciner at $2.45 \mathrm{GHz}$. Apart from the experimental investigations, the study also involved microwave analysis to approximately predict the mode numbers corresponding to specific solutions of the characteristic field equations in
\end{abstract}

*School of Mathematical Sciences, Queensland University of Technology, Brisbane, Australia. mailto:v.vegh@qut.edu.au

†Queensland University of Technology. mailto:i.turner@qut.edu.au

$\ddagger$ Australian Nuclear Science and Technology Organisation, Sydney, Australia. mailto: eas@ansto.gov . au

$\S$ Australian Nuclear Science and Technology Organisation

See http://anziamj.austms.org.au/V45/CTAC2003/Vegh/home.html for this article, (c) Austral. Mathematical Soc. 2004. Published February 17, 2004. ISSN 1446-8735 
the cavity as a function of powder bed height. The calculations reveal the dominant modes expected to propagate in the calciner. Here, a finite-volume time-domain technique simulates the electromagnetic phenomena inside the empty and loaded calciner. Because of the geometry of the calciner, special stair-case type boundary conditions ensure accurate calculations of the fields at the cylindrical conducting walls of the cavity. The simulation results confirm the experimentally observed mode structure inside the calciner. Thus the numerical technique can capture the nature of the modes in the case when the calciner is empty and loaded with a ceramic powder.

\section{Contents}

1 Introduction

2 Mathematical model

3 Boundary condition treatment

3.1 Incident plane . . . . . . . . . . . . . . . . C40

3.2 Conducting walls . . . . . . . . . . . . . . C C41

3.3 Material interface . . . . . . . . . . . . . . C C43

3.4 Absorbing layers . . . . . . . . . . . . . . C44

4 Results and discussion $\quad$ C45

5 Conclusions and future work $\quad$ C47

References

C48 


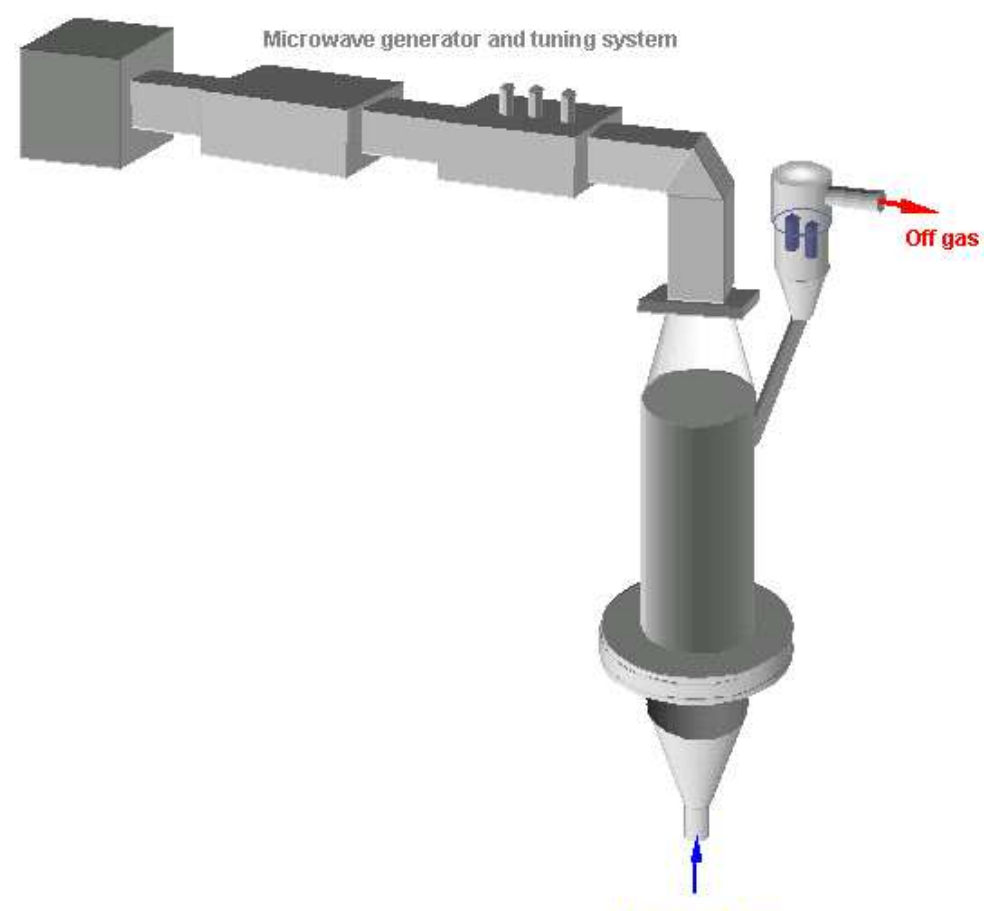

Fluidisation gas

Figure 1: The calciner and its components.

\section{Introduction}

For many years microwave technology has been a cheap source of energy in many different industrial applications [1]. More recently, specific applications related to fluidised beds have been of interest $[2,3]$. These types of processes have wide ranging applications not only in electromagnetics, but also in fluid dynamics.

In this paper, a prototype fluidised bed calciner has been constructed from a $405 \mathrm{~mm}$ long stainless steel pipe with $108 \mathrm{~mm}$ inside diameter (see 
Figure 1). The calciner is used to investigate heating of titanate based precursors and $\mathrm{SiC}$ powders. The system that delivers the electromagnetic energy to the fluidised bed was built to provide variable power between 0.6 and $6 \mathrm{~kW}$. This power is delivered through the top end of the calciner at $2.45 \mathrm{GHz}$. Microwaves were excited by a WR340 TE10 mode waveguide. Microwave reflections from the calcination cavity and the fluidised powder bed were measured for different titanate precursors and $\mathrm{SiC}$ powder as a function of bed height. Apart from the experimental investigations, the study also involved microwave analysis to approximately predict the mode numbers corresponding to specific solutions of the characteristic field equations in the cavity as a function of powder bed height. Maps of TE11p oscillation modes for the cavity were generated for an empty and powder loaded cavity, in the case of a Transverse Electric (TE) field with a 11 mode in the $x$ and $y$-coordinate directions, and $p$ modes in the longitudinal, or $z$-coordinate direction.

A numerical finite-volume time-domain technique (FV-TD) [4] was implemented to simulate the electromagnetic phenomena inside the empty and loaded calciner shown in Figure 1. Although in the past high order methods have been investigated [5, e.g.], here we use second order schemes as they are adequately capable of obtaining the nature of the solution.

\section{Mathematical model}

The Maxwell's equations [6] that govern the electromagnetic phenomena evolving in this configuration are

$$
\nabla \times \vec{E}=-\frac{\partial \vec{B}}{\partial t}, \quad \nabla \times \vec{H}=\frac{\partial \vec{D}}{\partial t}+\vec{J} .
$$

In equation (1), $\vec{E}$ and $\vec{H}$ are respectively the electric and magnetic field intensities, $\vec{D}$ and $\vec{B}$ are the electric and magnetic flux densities. The con- 

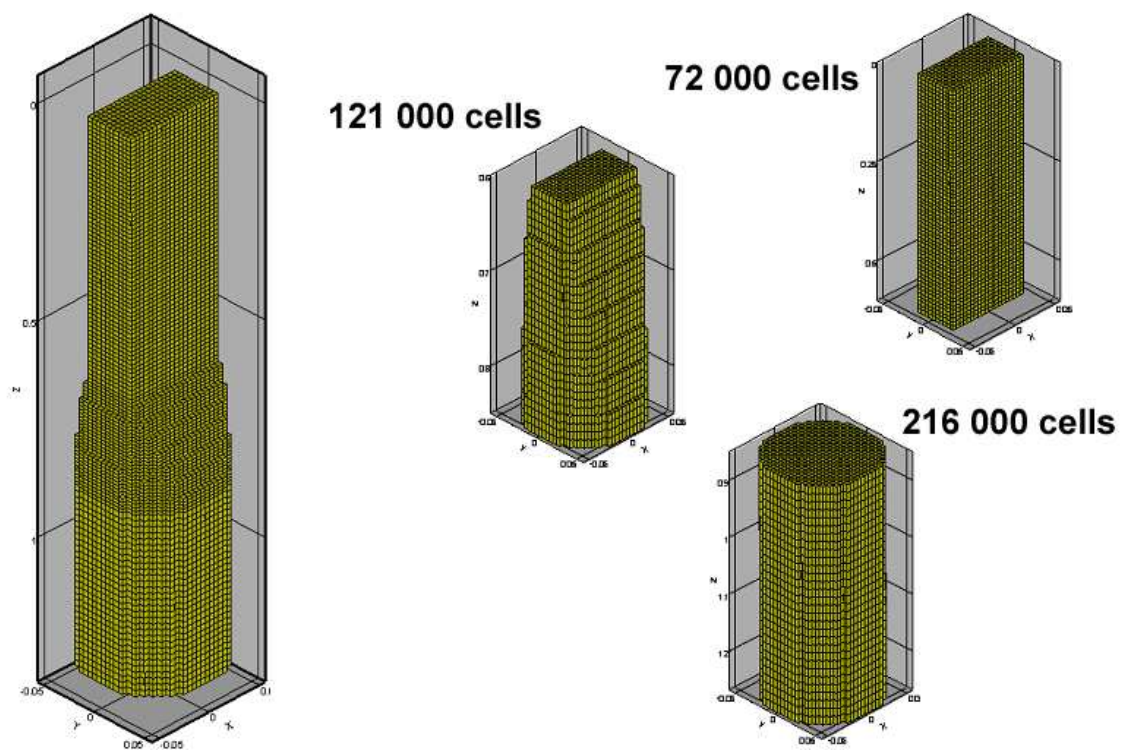

Figure 2: A coarse mesh that shows the stair-casing around the edges. 
stitutive relations are

$$
\vec{D}=\varepsilon \vec{E}, \quad \vec{B}=\mu_{0} \vec{H}, \quad \vec{J}=\sigma \vec{E}, \quad \varepsilon=\varepsilon_{0} \varepsilon^{\prime}, \quad \sigma=\omega \varepsilon_{0} \varepsilon^{\prime \prime},
$$

where $\mu_{0}$ is the magnetic permittivity, or permeability, of free-space, $\varepsilon_{0}$ is the electric permittivity and $\sigma$ is the conductivity. The conductivity is a measure of the material's ability to absorb electromagnetic energy, and is equal to $\sigma=\omega \varepsilon_{0} \varepsilon^{\prime \prime}$, where $\omega=2 \pi f$ and $f$ is the frequency. Usually for dielectric materials, the relative permittivity in complex form is written as $\varepsilon_{r}=\varepsilon^{\prime}-i \varepsilon^{\prime \prime}$, where $\varepsilon^{\prime}$ is the relative dielectric constant and $\varepsilon^{\prime \prime}$ is the relative loss factor. In this work a Cartesian mesh is used to discretise the rectangular waveguide and a stair-case mesh is used to mesh the oversized cylindrical chamber (see Figure 2). The finite-volume discretisation is constructed by integrating Maxwell's equations over a discrete cell located in the mesh [4]:

$$
\frac{\partial \vec{H}_{p}}{\partial t}=\frac{1}{\mu_{0} \delta_{v}} \sum_{\Gamma \in \zeta_{p}} \vec{n} \times \vec{E}_{\Gamma} \delta_{s}, \quad \frac{\partial \vec{E}_{p}}{\partial t}=\frac{1}{\varepsilon \delta_{v}} \sum_{\Gamma \in \zeta_{p}} \vec{n} \times \vec{H}_{\Gamma} \delta_{s}-\frac{\omega \varepsilon^{\prime \prime}}{\varepsilon^{\prime}} \vec{E}_{p} .
$$

where $p$ is the location at the cell centre at which the electric and magnetic field unknowns are stored. $\Gamma$ is a particular cell face of a finite-volume cell, which comprises faces $\zeta_{p}$, and $\vec{n}$ is the unit outward normal to face $\Gamma$. Equation (3) is solved using either one of the classical ODE solvers for the partial time derivative, or some other time discretisation technique is applied. For this investigation a leapfrog in time discretisation is used to resolve the partial time derivatives, which has been found previously [4] to provide time efficiency compared to high-order time discretisation techniques like RK3 and RK4. For a second order time discretisation and, as an illustration, taking only the first component of both the electric and magnetic fields, the leapfrog scheme is

$$
\left(\frac{\partial E_{x}}{\partial t}\right)_{p} \simeq \frac{\left(E_{x}\right)_{p}^{n+1}-\left(E_{x}\right)_{p}^{n}}{\delta_{t}}, \quad\left(\frac{\partial H_{x}}{\partial t}\right)_{p} \simeq \frac{\left(H_{x}\right)_{p}^{n+\frac{1}{2}}-\left(H_{x}\right)_{p}^{n-\frac{1}{2}}}{\delta_{t}}
$$

where $\delta_{t}$ is the time step of the finite-volume time-domain numerical solver. The representations for the $y$ and $z$ components of the fields are also cast 
in a similar form to (4). Consequently, equation (3) is now resolved in time using (4), and hence, the finite-volume time-domain discretisation for a cell is calculated as:

$$
\begin{aligned}
\vec{H}_{p}^{n+\frac{1}{2}} & =\vec{H}_{p}^{n-\frac{1}{2}}-\frac{\delta_{t}}{\mu_{0} \delta_{v}} \sum_{\Gamma \in \zeta_{p}} \vec{n} \times \vec{E}_{\Gamma}^{n} \delta_{s} \\
\vec{E}_{p}^{n+1} & =\frac{2 \varepsilon-\sigma \delta_{t}}{2 \varepsilon+\sigma \delta_{t}} \vec{E}_{p}^{n}+\frac{2 \delta_{t}}{\left(2 \varepsilon+\sigma \delta_{t}\right) \delta_{v}} \sum_{\Gamma \in \zeta_{p}} \vec{n} \times \vec{H}_{\Gamma}^{n+\frac{1}{2}} \delta_{s} .
\end{aligned}
$$

Obtain equations (5) and (6) by substituting (4) into (3), and rearranging respectively for the magnetic and electric fields at the highest time level, which formulates the discrete point form of the time-marching algorithm.

\section{Boundary condition treatment}

In this section, the treatment of the boundary conditions for the incident plane, conducting walls, material interface and absorbing layers are briefly elucidated.

\subsection{Incident plane}

The apparatus in Figure 1 is excited using a rectangular waveguide attachment, which generates $T E_{10}$ incident modes that propagate into the calciner. The generated modes have a specific nature and are captured using the $y$ component of the electric field, and the $x$-component and $z$-component of 
the magnetic field. The nature of these fields are

$$
\begin{aligned}
& E_{y}=E_{0} \sin \left(\frac{\pi x}{a}\right) \cos \left(\omega t-\beta_{0} z_{0}\right), \\
& H_{x}=-\frac{\beta_{0}}{\omega \mu_{0}} E_{0} \sin \left(\frac{\pi x}{a}\right) \cos \left(\omega t-\beta_{0} z_{0}\right), \\
& H_{z}=-\frac{\pi}{\omega \mu_{0} a} E_{0} \cos \left(\frac{\pi x}{a}\right) \sin \left(\omega t-\beta_{0} z_{0}\right),
\end{aligned}
$$

where

$$
E_{0}=2 \sqrt{\frac{P_{0}}{a b \varepsilon_{0}}}, \quad \beta_{0}=\sqrt{\omega^{2} \mu_{0} \varepsilon_{0}-\left(\frac{\pi}{a}\right)^{2}} .
$$

In equation (7), $P_{0}$ is the average input power of the waveguide, $z_{0}$ is the location of the plane of incidence, and $a$ and $b$ are the cross-sectional dimensions of the waveguide in the $x$ and $y$ directions, respectively.

\subsection{Conducting walls}

Conducting wall boundary conditions for the electric and magnetic fields are

$$
\vec{n} \times \vec{E}=\overrightarrow{0}, \quad \vec{n} \cdot \vec{H}=0,
$$

where $\vec{n}$ is assumed to be the unit normal vector pointing outwards from the domain of the apparatus. Effectively, (8) implies that the tangential components of the electric field and the normal components of the magnetic field are zero on the conducting walls. For an apparatus that has rectangular sides the implementation of these boundary conditions is straightforward [4]. However, in this work, since a regular Cartesian mesh was used for the discretisation of the whole domain, special care was taken at curved surfaces. A stair-case approach was used to capture the behaviour of the electric and magnetic fields at the conducting walls of the cylinder. The adaptation of the stair-case boundary treatment for the finite-volume time-domain method proposed here appears novel. 


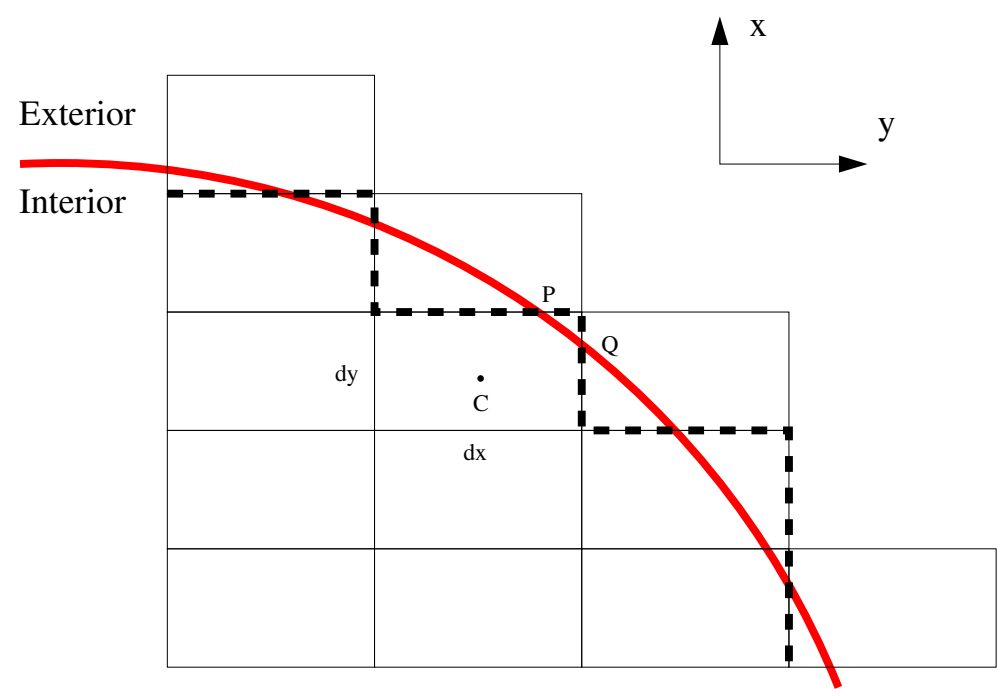

FiguRE 3: The stair-case mesh to approximate a curved surface. The bold line depicts the outline of the calciner wall and the dashed line illustrates the cell used in the numerical scheme. 
Figure 3 illustrates the mesh at the curved surfaces of the calciner. Since for the FV-TD method, see equations (5) and (6), the unknowns are stored at the cell centres, any cell centres that lie outside of the curved surfaces are subsequently not considered in the numerical scheme. Volumes of cells $\delta_{v}$ are appropriately adjusted for use in the FV-TD equations. The axes were chosen such that the $z$-coordinate was aligned with the longitudinal dimension of the calciner. Also, the origin was located at the centre of the plane of incidence. This allowed for easy approximation of the volumes of the cells required in the FV-TD method at the edge of the calciner. For Figure 3 assume that $C$ is at location $(x, y)$ and $P$ and $Q$ are needed to calculate the area as $d x \times d y / \lambda$. From this area, the volume of the cell is $\delta_{v}=d x \times d y \times d z / \lambda$, where $\lambda$ is the factor that removes the area from each cell that lies outside of the curve with radius $r$. If $P$ and $Q$ are known, then $\lambda$ is calculated using vector algebra.

\subsection{Material interface}

At material interfaces the dielectric properties change, and this change in the electromagnetic behaviour at the interface is governed by:

$$
\begin{aligned}
\vec{n} \times\left(E_{m}-E_{a}\right) & =\overrightarrow{0}, \\
\vec{n} \cdot\left(\vec{D}_{m}-\vec{D}_{a}\right) & =0, \\
\vec{n} \times\left(\vec{H}_{m}-\vec{H}_{a}\right) & =\overrightarrow{0}, \\
\vec{n} \cdot\left(\vec{H}_{m}-\vec{H}_{a}\right) & =0,
\end{aligned}
$$

where $m$ is an adjacent cell unknown right at the material interface, and $a$ is the neighbouring unknown in free-space or air. From equation (9) it is evident that the electric field is discontinuous at a material interface, while the magnetic field is continuous. The implementation at the material interface is therefore not straightforward, and requires a special approximation to capture the electric field propagation and reflection at the boundary. 
Using adjacent cells to the material interface, a second order Taylor approximation finds the unknowns right on the face of the material. For the purposes of providing an accurate approximation for the facial value of the electric field on an interface the following new formulation that uses the assumption of plane wave behaviour near an interface is made:

$$
\left(E_{u}\right)_{p+\frac{1}{2}}=\frac{\beta_{m}}{\beta_{a}+\beta_{m}}\left(E_{u}\right)_{p}+\frac{\beta_{a}}{\beta_{a}+\beta_{m}}\left(E_{u}\right)_{p+1},
$$

where

$$
\beta_{i} \propto \sqrt{\varepsilon_{i}^{\prime}\left[1+\sqrt{1+\left(\varepsilon_{i}^{\prime \prime} / \varepsilon_{i}^{\prime}\right)}\right]}, \quad i=a, m .
$$

\subsection{Absorbing layers}

When propagating electromagnetic waves into waveguides and cavities, some of the fields will be absorbed and others will be reflected. These reflected waves propagate back up to the waveguide. Consequently, arrangements in the numerical algorithm must be made to absorb these reflected electric and magnetic field components from interfering with the phase and amplitude of the incident wave. We adopt the perfectly matched layer discussed in [7]. To achieve this, Maxwell's equations are rewritten in a different form so that the impedance of free-space is matched to the impedance of the layer introduced to remove these unwanted electromagnetic waves [7]. The new equations are discretised using the finite-volume strategy [4] to obtain the following point 
representations inside the absorption layer:

$$
\begin{aligned}
\vec{E}_{p}^{n+1} & =\vec{E}_{p}^{n}+\left(\frac{2 \varepsilon \delta_{t}+\sigma \delta_{t}^{2}}{2 \varepsilon^{2} \delta_{v}}\right) \sum_{\Gamma \in \zeta_{p}} \vec{n} \times \vec{H}_{\Gamma}^{n+\frac{1}{2}} \delta_{s}+\frac{\sigma \delta_{t}^{2}}{\varepsilon^{2} \delta_{v}} \vec{F}_{p}^{n-\frac{1}{2}}, \\
\vec{F}_{p}^{n+\frac{1}{2}} & =\vec{F}_{p}^{n-\frac{1}{2}}+\sum_{\Gamma \in \zeta_{p}} \vec{n} \times \vec{H}_{\Gamma}^{n+\frac{1}{2}} \delta_{s} \\
\vec{H}_{p}^{n+\frac{1}{2}} & =\vec{H}_{p}^{n-\frac{1}{2}}-\left(\frac{2 \mu_{0} \delta_{t}+\bar{\sigma} \delta_{t}^{2}}{2 \mu_{0} \delta_{v}}\right) \sum_{\Gamma \in \zeta_{p}} \vec{n} \times \vec{E}_{\Gamma}^{n} \delta_{s}-\frac{\bar{\sigma} \delta_{t}^{2}}{\mu_{0}^{2} \delta_{v}} \vec{G}_{p}^{n-1}, \\
\vec{G}_{p}^{n+1} & =\vec{G}_{p}^{n-1}+\sum_{\Gamma \in \zeta_{p}} \vec{n} \times \vec{E}_{\Gamma}^{n} \delta_{s} .
\end{aligned}
$$

Note that only the components of the fields in the propagation direction (that is, normal components) are treated with (11) and (12); all other fields (that is, tangential components) are still governed by (5) and (6). The choice of $\sigma$ for the material is such that as the waves propagate inside the material the conductivity of the material increases.

\section{Results and discussion}

This work involved the modelling of the calciner, which is an apparatus that delivers electromagnetic energy to a material loaded at the bottom of the cylindrical chamber (see Figure 1). Figure 4 depicts the solution of the time averaged electric field inside the calciner. In the figure, the wider section is of most importance, which corresponds to the cylindrical chamber. The apparatus was discretised into approximately 410000 Cartesian cells, however to exhibit the nature of the mesh and to provide insight into how the domain was decomposed, a coarse mesh is shown in Figure 2.

Figure 4 illustrates the results for both the empty and loaded chambers. From the results it is evident that the mode structure in the empty chamber 


\section{Empty chamber}

\section{Loaded chamber}
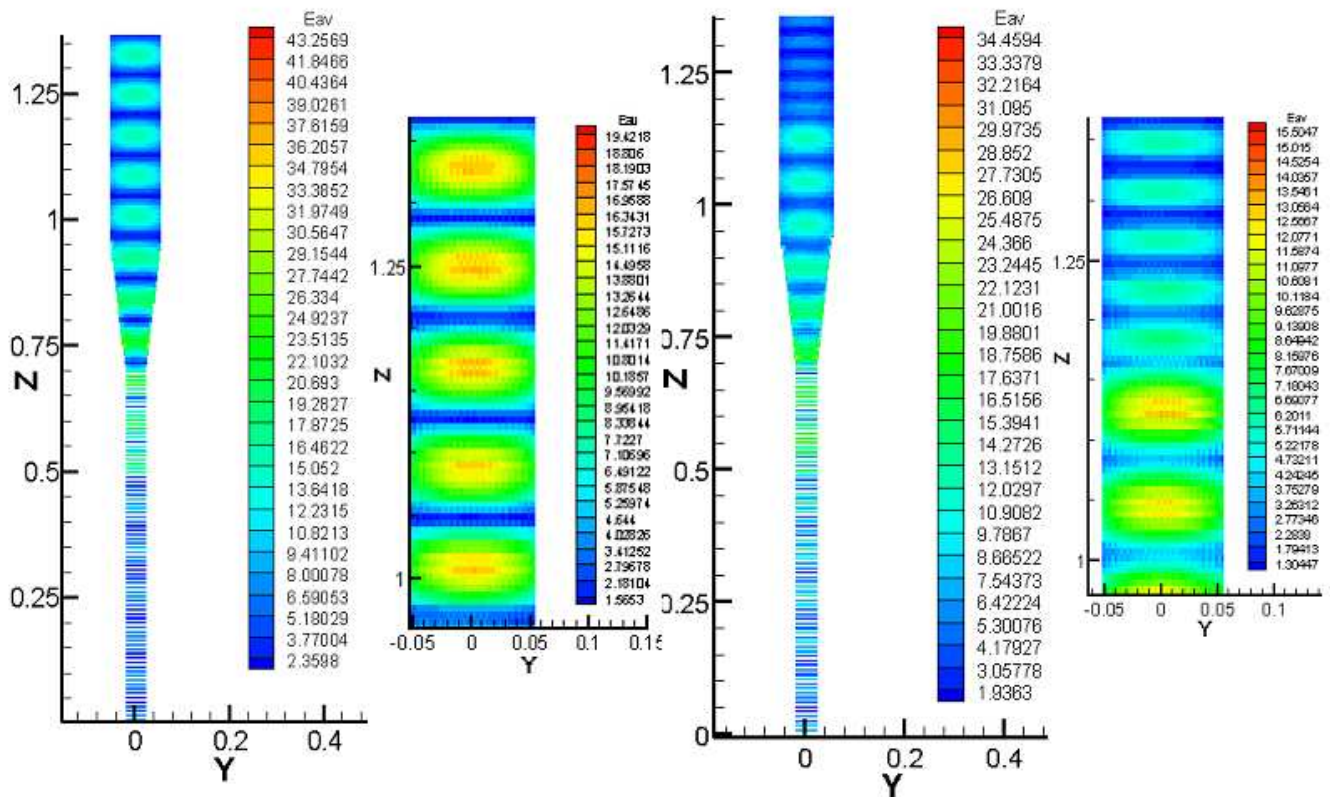

FigURE 4: TE11p numerical results for the empty and loaded chamber. 
resembles the $T E_{115}$ mode as predicted analytically and confirmed from the maps of the oscillation modes. When the chamber is loaded with the ceramic powders, the mode observed in the cylindrical section of the chamber is changed to $T E_{118}$ for a material with dielectric property $\varepsilon_{r}=2.54-0.15 j$ and bed height of $0.3 \mathrm{~m}$. This change is a result of the reduced wavelength inside the material due to the material's properties. The result obtained for the loaded chamber also corresponds to the experimental observations.

\section{Conclusions and future work}

These preliminary investigations considered the prototype calciner used for the purpose of nuclear waste treatment at the Australian Nuclear Science and Technology Organisation. We focused on the development of a numerical algorithm to model the behaviour of the electromagnetic fields within the heating apparatus or calciner.

The domain of the problem was discretised using a regular mesh with some special boundary condition implementation to capture the curvature of the apparatus. An existing finite-volume time-domain method [4] was used with a stair-case mesh to approximate the unknowns at cylindrical conducting boundary walls. The algorithm predicted the nature of the fields within the calciner both in the case when there is no material present, and also in the case when the calciner is loaded with ceramic powders. Solutions from the numerical algorithm verified the observed $T E_{115}$ and $T E_{118}$ modes for an empty and loaded calciner, respectively.

Future work will see the investigation of reflections within the calciner, which focuses on modelling the heat distribution inside the material and how this heating affects the reflections that occur at the material interface interacting with the evolving electromagnetic fields. 
Acknowledgements: The authors from QUT thank the authors from ANSTO for their significant contribution towards this paper in the form of extensive experimental results. The QUT authors also acknowledge the QUT high performance computing team for many fruitful discussions and the provision of the supercomputing facilities.

\section{References}

[1] A. C. Metaxas and R. J. Meredith. Industrial Microwave Heating. Peter Peregrinus Limited, London, UK, 1983. C36

[2] A. Baysar. Microwave heating applications of fluidized beds: High-purity silicon production. PhD Thesis, Arizona State University, 1992. C36

[3] G. Roussy, S. Jassm and J-M. Thiebaut. Modeling of a fluidized bed irradiated by a single or a multimode electric microwave field distribution, Journal of microwave power and electromagnetic energy, 30(3):178-187, 1995 C36

[4] V. Vegh. Numerical Modelling of Industrial Microwave Heating. Queensland University of Technology, PhD thesis, 2003. C37, C39, C41, C44, C47

[5] J. S. Shang. High-Order Compact Difference Schemes for Time-Dependent Maxwell Equations. Journal of Computational Physics, 153:312-333, 1999. C37

[6] R. E. Collin. Field Theory of Guided Waves McGraw-Hill Book Company, 1960. C37

[7] P. G. Petropoulos, L. Zhao and A. C. Cangellaris. A Reflectionless Sponge Layer Absorbing Boundary Condition for the Solution of 
Maxwell's Equations with High-Order Staggered Finite Difference Schemes. Journal of Computational Physics, 139:184-208, 1998. C44 